OPEN ACCESS

Edited by:

Jean-christophe Augustin,

École Nationale Vétérinaire d'Alfort,

France

Reviewed by:

Lei Dai,

lowa State University, United States Giorgia Perpetuini,

Università degli Studi di Teramo, Italy

${ }^{*}$ Correspondence:

Jinlin Huang

jinlin@yzu.edu.cn

Specialty section:

This article was submitted to

Food Microbiology,

a section of the journal

Frontiers in Microbiology

Received: 03 December 2017

Accepted: 27 July 2018

Published: 15 August 2018

Citation:

Du X, Kong K, Tang H, Tang H, Jiao X and Huang J (2018) The Novel Protein Cj0371 Inhibits Chemotaxis

of Campylobacter jejuni.

Front. Microbiol. 9:1904.

doi: 10.3389/fmicb.2018.01904

\section{The Novel Protein Cj0371 Inhibits Chemotaxis of Campylobacter jejuni}

\author{
Xueqing Du, Ke Kong, Hong Tang, Haiyan Tang, Xinan Jiao and Jinlin Huang* \\ Jiangsu Key Lab of Zoonosis, Jiangsu Co-Innovation Center for Prevention and Control of Important Animal Infectious \\ Diseases and Zoonoses, College of Bioscience and Biotechnology, Yangzhou University, Yangzhou, China
}

cj0371 is a novel gene that is associated with Campylobacter jejuni virulence, and an isogenic mutant of cj0371 showed hyper chemotaxis and motility. Chemotactic motility is an important virulence factor and is involved in C. jejuni pathogenesis. Campylobacter sp. has specific variations of the common chemotaxis components, including histidine autokinase CheA, coupling scaffold protein CheV, chemotaxis response regulator protein $\mathrm{CheY}$ and several chemoreceptor proteins. In this study, we used immunoprecipitation combined with LC-MS/MS analyses to screen six chemotaxis pathway proteins that potentially interact with the putative protein Cj0371. qRT-PCR was used to quantitatively analyze the expression of these chemotaxis genes and basic flagella genes. The results showed that the expression of cheV, cj1110c, and cj0262c was significantly up-regulated, and four flagella genes also had up-regulated expression in the cj0371 mutant. GST pull-down analyses found that Cj0371 interacted with the receiver domain of the CheV protein. Enzyme-coupled spectrophotometric assays showed that the ATPase activity of CheA was higher when Cj0371 was not present in the chemotaxis reaction medium. Therefore, we concludes that cj0371 has a negative influence on $C$. jejuni chemotaxis, which may occur by adjusting the receiver domain of $\mathrm{CheV}$ to influence chemotaxis. This paper provides a new component in the chemotaxis pathway of $C$. jejuni for the first time and highlight the complexity of this remarkable pathway.

Keywords: Campylobacter jejuni, Cj0371 protein, protein interaction, chemotaxis protein, chemotaxis pathway

\section{INTRODUCTION}

Campylobacter jejuni is the causative agent of acute bacterial gastroenteritis in humans (Hoang et al., 2011). In the past decade, human infections with the zoonotic pathogen have progressively risen in developed as well as developing countries (Bereswill et al., 2016; Berradre-Saenz et al., 2017). According to previous data, more than $80 \%$ of confirmed cases of campylobacteriosis were reported to be associated to Campylobacter jejuni (Helwigh et al., 2015). The clinical manifestation of campylobacteriosis is severe gastroenteritis (Bronnec et al., 2016). Infected individuals may be asymptomatic or exhibit rather mild symptoms like watery diarrhea, whereas other patients suffer from acute ulcerative enterocolitis with inflammatory bloody diarrhea. On rare occasions, reactive arthritis or neurological complications including Guillain-Barré and Miller-Fisher syndromes may arise with a latency of several weeks to several months postinfection (Wakerley et al., 2014).

The recognized pathogenic mechanisms of $C$. jejuni, including motility, chemotaxis, host cell adhesion and invasion, as well as the production of toxin, are mediated by several virulence factors 
(Flanagan et al., 2009). Chemotactic motility is an important virulence factor, it is the ability of bacterial cells to detect temporal changes in the chemical concentration of their surrounding environment. Flagella-mediated motility have been reported to play an important role in the intestinal colonization of avian and the invasion of intestinal epithelial cells (Nachamkin et al., 1993; Szymanski et al., 1995). Campylobacter sp. has specific variations of the common chemotaxis components, including histidine autokinase CheA, coupling scaffold protein $\mathrm{CheV}$, chemotaxis response regulator protein $\mathrm{CheY}$ and several chemoreceptor proteins such as $\mathrm{Cj0019c}, \mathrm{Cj} 1564, \mathrm{Cj} 0262 c$, and $\mathrm{Cj} 1110 \mathrm{c}$. In our previous research, we identified a novel gene cj0371 and found it influences the invasion and colonization of C. jejuni, so we discerned that cj0371 is a virulence-associated gene. In addition, we also confirmed that cj0371 plays a negative role in motility and chemotaxis. We hypothesize that cj0371 is a new virulence-associated gene and, by influencing chemotaxis, plays a negative role in $C$. jejuni pathogenicity ( $\mathrm{Du}$ et al., 2016).

In this paper, we continue to explore the function of Cj0371 protein. First, we carried out co-immunoprecipitation (co-IP) coupled to LC-MS/MS analysis to discover the proteins that interact with Cj0371. Then, we selected chemotaxis pathway proteins as one of the emphases for research, and used qRTPCR to quantify the expression level of these possible interactive chemotaxis proteins. We used GST pull-downs to validate predicted protein-protein interactions. Finally, we used enzymecoupled spectrophotometric assays to detect the ATPase activity of CheA when Cj0371 protein was added to the chemotaxis reaction medium. Our study presents an insight into the pathogenic mechanism of C. jejuni and offers exciting new directions for future research.

\section{MATERIALS AND METHODS}

\section{Bacterial Strains, Media, and Culture Conditions}

The complete list of bacterial strains and plasmids used in this study are described in Table 1. C. jejuni $\Delta$ cj0371 and C. jejuni $\Delta c j 0371+$ strains were described in our previous report (Du et al., 2016). E. coli DE3 expressing GST-Cj0371 and $6 \times$ HisCj0371 protein were preserved in our laboratory. C. jejuni strains were routinely grown on Campy blood-free selective medium (CCDA; Oxid Ltd., United Kingdom) or MuellerHinton agar (MH; BD Ltd., United States) microaerobically [85\% $\mathrm{N}_{2}(\mathrm{v} / \mathrm{v}), 10 \% \mathrm{CO} 2(\mathrm{v} / \mathrm{v})$, and $\left.5 \% \mathrm{O}_{2}(\mathrm{v} / \mathrm{v})\right]$ in a jar at $42^{\circ} \mathrm{C}$. E. coli DH5 $\alpha$ and DE3 (BL21) that were used for cloning and expressing $C$. jejuni proteins were routinely cultured in LuriaBertani (LB) agar plates at $37^{\circ} \mathrm{C}$ overnight. When required, $\mathrm{LB}$ broth was supplemented with ampicillin $(100 \mu \mathrm{g} / \mathrm{mL})$, kanamycin $(50 \mu \mathrm{g} / \mathrm{mL})$ and chloramphenicol $(30 \mu \mathrm{g} / \mathrm{mL})$. The C. jejuni transformants were selected on plates supplemented with $50 \mu \mathrm{g} / \mathrm{mL}$ kanamycin and/or $20 \mu \mathrm{g} / \mathrm{mL}$ chloramphenicol, and all C. jejuni strains were stored at $-70^{\circ} \mathrm{C}$ in brain heart infusion broth (BHI; BD Ltd., United States) containing 30\% glycerol.
TABLE 1 | Bacterial strains and plasmids used in this study.

\begin{tabular}{|c|c|c|}
\hline Strains or plasmids & Genotype & $\begin{array}{l}\text { Source or } \\
\text { reference }\end{array}$ \\
\hline \multicolumn{3}{|l|}{ Strains } \\
\hline \multicolumn{3}{|l|}{ E. coli } \\
\hline $\mathrm{DH} 5 \alpha$ & 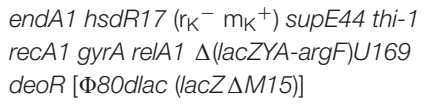 & Invitrogen \\
\hline BL21 (DE3) & F- ompT hsdSB (rB- mB-) gal dcm & Novagen \\
\hline DE3 (GST) & BL21(DE3) (pGEX-6P-l) & This study \\
\hline DE3 (GST-Cj0371) & BL21(DE3) (pGEX-6P-I-cj0371) & This study \\
\hline DE3 (His-Cj0371) & BL21(DE3) (pET-30a-cj0371) & This study \\
\hline \multicolumn{3}{|l|}{ C. jejuni } \\
\hline C. jejuni 11168 & C. jejuni NCTC11168 & This study \\
\hline C. jejuni $\Delta c j 0371$ & C. jejuni NCTC11168 cj0371:: $\mathrm{cm}^{r}$ & This study \\
\hline C. jejuni $\Delta c j 0371+$ & $\begin{array}{l}\text { C. jejuni NCTC11168 cj0371:: } \mathrm{cm}^{r} \\
\text { (pRY107-PmetK-flag-cj0371) }\end{array}$ & This study \\
\hline
\end{tabular}

Plasmids

pET-19b

Expressing His-tagged vector, AmpR

Novagen

\section{Immunoprecipitation of Interacting Proteins and LC-MS/MS Analysis}

The C. jejuni strain expressing a FLAG-Cj0371 protein was constructed as descried in our previous study (Du et al., 2016). The steps were identical to the complementation of the cj0371 mutation in C. jejuni $\Delta$ cj0371. The only difference was the use of different specific primers; the flag sequence was added by the forward primer in this study. The C. jejuni strain expressing a FLAG-Cj0371 protein was grown on two Mueller-Hinton agar plates for approximately $24 \mathrm{~h}$, resuspended in PBS, pelleted at 5,000 rpm, and then resuspended in $2 \mathrm{~mL}$ of lysis buffer (50 mM Tris $\mathrm{HCl}, \mathrm{PH} 7.4,150 \mathrm{mM} \mathrm{NaCl}, 1 \mathrm{mM}$ EDTA, $1 \%$ Triton $\mathrm{X}-100$ ), $1 \%$ Triton $\mathrm{X}-100$, and protease inhibitors (ROCHE). After lysis by sonication, cell debris was removed by centrifugation at $12,000 \mathrm{rpm}$, supernatants were recovered, and immunoprecipitation of FLAG-Cj0371 protein was performed using anti-FLAG M2 affinity gel following the manufacturer's recommendations and methods described in a previous report (Gao et al., 2014). In brief, the ANTI-FLAG M2 affinity gel was thoroughly suspended in the vial to achieve a uniform suspension of the resin. A $40 \mu \mathrm{L}$ volume of the gel suspension was transferred to a $1.5 \mathrm{~mL}$ tube. The resin was centrifuged at $5,000 \times g$ for $30 \mathrm{~s}$. The supernatant was removed and the resin was washed three times with TBS (50 mM Tris- $\mathrm{HCl}, 150 \mathrm{mM} \mathrm{NaCl}, \mathrm{pH}$ 7.4). Then, $200 \mu \mathrm{L}$ of cell lysate was added to the washed resin and brought to a final volume of $700 \mu \mathrm{L}$ by the addition of lysis buffer. All samples were shaken for $3 \mathrm{~h}$ at $4^{\circ} \mathrm{C}$. After incubation, the resin was washed six times with $0.5 \mathrm{~mL}$ of TBS and eluted with $100 \mu \mathrm{L}$ of $0.1 \mathrm{M}$ glycine $\mathrm{HCl}, \mathrm{PH} 3.5$. All elution products were pooled and loaded onto $12 \%$ SDS-PAGE gel, and the gel pieces was sent to Protech in Suzhou for LC-MS/MS.

\section{RNA Preparation and Real-Time PCR}

The wild type C. jejuni 11168 and the previously described line C. jejuni $\Delta c j 0371$ were grown in Mueller-Hinton broth 
(MH, BD, United States) under microaerobic conditions at $42^{\circ} \mathrm{C}$ with shaking $(180 \mathrm{rpm})$ and harvested at the stationary $(18 \mathrm{~h})$ phase of growth. C. jejuni bacteria pellets were resuspended in RNAprotect Bacteria Reagent (Qiagen) to preserve them. For RNA preparation, bacteria pellets were resuspended in Qiagen RNeasy lysis buffer containing $\beta$-mercaptoethanol and shocked in a vortex for $30 \mathrm{~s}$. The RNA was further purified using the Qiagen RNeasy Plus mini kit as described previously and in the manufacturer's instructions (Chandrashekhar et al., 2015). The absence of DNA was confirmed by PCR amplification using Campylobacter-specific 16S rRNA primers, and total RNA was quantified in a NanoDrop 1000 device (Thermo Scientific, Germany). cDNA was prepared from equivalent amounts $(1 \mu \mathrm{g})$ of total RNA using the PrimeScript ${ }^{\text {TM }}$ RT-reagent kit with gDNA Eraser (Takara) according to the manufacturer's instructions.

Quantitative RT-PCR was performed using SYBR green mix (Roche) on a $2 \mu \mathrm{L}$ volume of cDNA and normalized to the results of a corresponding 16S rRNA gene control reaction mixture prepared from the same cDNAs (primers used for RT-PCR are listed in Supplementary Tables S1, S2). Reactions were carried out in a 7500 Real Time PCR system [Applied Biosystems (ABI, United States)]. Cycling conditions were as follows: denaturation for $10 \mathrm{~min}$ at $95^{\circ} \mathrm{C}$ and amplification for 40 cycles at $95^{\circ} \mathrm{C}$ for $10 \mathrm{~s}, 59^{\circ} \mathrm{C}$ for $30 \mathrm{~s}$, and $72^{\circ} \mathrm{C}$ for $30 \mathrm{~s}$. For DNA quantification, a serial standard was prepared from a purified PCR product of the gene of interest. Each reaction was performed in triplicate. Samples were tested in triplicate, and the analysis was replicated at least three times. Relative gene expression was determined by the $2^{-\Delta \Delta C_{\mathrm{T}}}$ method (Livak and Schmittgen, 2001).

\section{Recombinant Expression and Purification of $\mathbf{C}$. jejuni Proteins}

CheA, CheY, CheV, Cj1110c, Cj1564, Cj0262c, and Cj6462 proteins were expressed by the pET-19b plasmid in the DE3 system. We used a one-step cloning kit to generate the recombinant expressing plasmids following the manufacturer's recommendations and methods described in a previous report. The genes cheA, cheY, cheV, cj1110c, cj1564, cj0262c, and cj1564 were amplified by PCR from C. jejuni 11168 using specific primers which had homologous sequence with $\mathrm{pET}-19 \mathrm{~b}$, with the restriction sites Nde I in the forward primers and $\mathrm{BamH}$ I in the reverse primers (Supplementary Table S3). Then, the fragments were cloned into pET-19b using one-step cloning kit. The recombinant plasmids were used to transform E. coli $\mathrm{DH} 5 \alpha$. Transconjugants were selected on plates containing Ampicillin $(100 \mu \mathrm{g} / \mu \mathrm{L})$. The positive transconjugants were confirmed by PCR analysis and nucleotide sequencing. The plasmids extracted from DH5 $\alpha$ were transferred into DE3 (BL21) to express.

To determine the expression of different proteins from individual clones, preliminary expression was carried out as described in previous reports (Suryanarayana et al., 2016; Faber et al., 2016). The recombinantly expressed $\mathrm{CheA}$, CheY, CheV, Cj0371, and Cj1564 (317-662 residues) proteins contained $6 \times$ His tags on their $\mathrm{N}$ termini and were purified from the soluble bacterial fraction by affinity chromatography using Ni2nitrilotriacetic acid (NTA)-Sepharose beads (Novagen) under native conditions. The purification procedure was modified according to the manufacturer's recommendations for the His Bind Purification Kit and methods described in a previous report (Novagen, Madison, WI, United States) (Sheng et al., 2007). The eluted proteins were analyzed by SDS-PAGE, pooled and dialyzed against at least three changes of PBS using a dialysis chamber (biosharp, $50 \mathrm{kDa}$ ).

\section{Anti-GST Tag Pull Down Assay}

As described in a previous report, to investigate protein interactions with Cj0371, GST pull-down analysis was performed (Vikis and Guan, 2004). A volume of $200 \mu \mathrm{L}$ of the soluble fractions of DE3 (pGEX-6P-I-cj0371) or DE3 (pGEX-6P-I) was transferred to mini spin columns as the bait protein and 300 $\mu \mathrm{L}$ PBST (add 1\% TritonX-100 to PBS) was added, then the columns were incubated for $3 \mathrm{~h}$ at $4^{\circ} \mathrm{C}$ with $50 \mu \mathrm{L}$ of Anti-GST beads (Thermo Scientific). Then, the resin was washed six times with $500 \mu \mathrm{L}$ PBST. After that, $200 \mu \mathrm{L}$ soluble fraction of DE3 (pET-19b-cheV) or other recombinant strains was added to the columns and incubated for $6 \mathrm{~h}$ at $4^{\circ} \mathrm{C}$. The resin was washed with PBST again approximately 10 times. Bound proteins were eluted with $60 \mu \mathrm{L} 10 \mathrm{mmol}$ glutathione. The eluted proteins were analyzed by Western blotting using the anti-His monoclonal antibody as the primary antibody.

\section{CheA Protein ATPase Activity Assay}

We investigated the effect of Cj0371 in the chemotaxis pathway using a coupled enzyme assay, which was based on a reaction in which the regeneration of hydrolyzed ATP is coupled to the oxidation of NADH (Sehgal et al., 2016). CheA ATPase activity was assayed using an enzyme-coupled spectrophotometric assay as described by previous reports with some modifications (Ninfa et al., 1988, 1991). The reaction was performed in $100 \mu \mathrm{L}$ of a basic medium containing: $100 \mathrm{mM} \mathrm{K}_{3} \mathrm{PO}_{4}, \mathrm{PH} 7.5,5 \mathrm{mM} \mathrm{Mgcl}_{2}$, $0.1 \mathrm{mM}$ EDTA, $0.1 \mathrm{mM}$ DTT, $10 \mathrm{mM}$ ATP, $0.2 \mathrm{mM} \mathrm{NADH}$, $1 \mathrm{mM}$ PEP, 2 U PK (Sigma), and 6.6 U L-LDH (Roche). The chemotaxis proteins mix volume was $100 \mu \mathrm{L}$, if one protein was not put in the mix, and brought to a final volume with deionized water. At the beginning of the reaction, the reaction mix was equilibrated at ambient temperature. The reaction was conducted at ambient temperature, and $\mathrm{NADH}$ oxidation was monitored in a Microplate Reader recording the absorbance at $340 \mathrm{~nm}$ every $30 \mathrm{~s}$. According to a previous report, we selected the chemotaxis proteins mix including $0.2 \mu \mathrm{M}$ CheA, $10 \mu \mathrm{M}$ CheY, $4 \mu \mathrm{M} \mathrm{CheV,}$ and $1 \mu \mathrm{M} \mathrm{Cj1110c}$ (Levit et al., 1998). The reactions were initiated by the addition of chemotaxis proteins mix, and the course of the reaction was monitored until a stable linear decay of absorbance was observed. The rate of decay over 15-30 min was used to indicate ATP hydrolysis rates. The control experiment was the reaction in the absence of any protein.

\section{Statistical Analysis}

Data analysis was performed using GraphPad. Statistical significance of the data was determined using Student's $t$-test in cases, where only two data sets were compared. The values of $P<0.05$ was considered statistically significant. 


\section{RESULTS}

\section{Cj0371 May Play a Role in Chemotaxis Pathway of C. jejuni}

Co-immunoprecipitation followed by liquid chromatographymass spectrometry (LC-MS/MS) analysis is a useful way to screen interacting proteins (Schieltz et al., 2006). We selected this strategy to screen for proteins that interact with Cj0371. The screened proteins were classified according to the pathway (KEGG Pathway Database). We screened approximately 18 proteins, 2 proteins in the two-component system, 6 proteins in the bacterial chemotaxis pathway (which we call chemotaxis proteins in this paper), 5 proteins in metabolic pathways (including glucose metabolism, nucleic acid metabolism, and protein metabolism), 2 proteins in RNA degradation, 2 proteins in the bacterial secretion system, and 1 flagella protein, FlgE (Table 2). However, not all the proteins identified by screening actually interact with Cj0371. A few proteins in same pathway were identified, that may have a cascade of interactions between proteins, and so we reasoned that Cj0371 may play a role in these pathways. According to our previous research, we found when cj0371 was inactivated, chemotaxis and motility of C. jejuni was increased. Therefore, in this paper we focused on the chemotaxis pathway of $C$. jejuni for further research.

\section{RT-qPCR Quantification of Chemotaxis and Flagella Genes}

RT-qPCR was used to confirm the differential expression of major chemotaxis pathway genes (including these chemotaxis genes that screened by co-IP) and the flagellar pathway genes that are regulated by chemotaxis. Whether in $\log$ (at $12 \mathrm{~h}$ of $C$. jejuni growth) or stationary phase $(24 \mathrm{~h}), c h e V, c j 1564, c j 0262 c$, and cj1110c all showed increased expression in C. jejuni $\Delta c j 0371$.
Especially, compared to the wild type C. jejuni 11168 , cheV had significantly increased expression in C. jejuni $\Delta c j 0371(12 \mathrm{~h}$, $P<0.05 ; 24$ h, $P<0.01$; Figures 1A,B). The flagellar motility of $C$. jejuni is controlled by chemotaxis. The transition between counterclockwise and clockwise rotation is controlled by proteins located at the base of the flagellar motor (Welch et al., 1993). Therefore, we also characterized flagellar base genes differential expression by RT-qPCR. These flagellar genes included the genes encoding flagellar hook ( $f l g E)$, C ring ( $f l i M)$, MS ring $(f l i F)$, P ring $(f l g I, f l g C)$, flagellar output system $(f h A, f h B)$ and flagellar motor protein (motA). In the $\log$ phase of $C$. jejuni growth, the flagellar genes $f l h A, f l h B, f l i M$, and $f l g C$ showed increased expression in the C. jejuni $\Delta c j 0371$ (Figures 1C,D). In stationary phase, it may be that cj0371 mutants enter the declining period earlier than the wild type strain.

\section{Interaction of Cj0371 Proteins With Screened Chemotaxis Proteins}

To provide additional evidence for a potential direct role of Cj0371 in chemotaxis, we searched for the interaction of chemotaxis proteins identified in our screen with Cj0371 protein. First, we used the DE3 (pET-19b) expression system to express 7 proteins or polypeptides including $\mathrm{CheA}, \mathrm{CheV}$, CheY, Cj1110c, Cj1564 (residues 42-290 of Cj1564), Cj0262c (residues 1-319 of Cj0262c) and Cj6462 (This paper named. It is the residues 317-662 of Cj1564, which are identical to residues 320-665 of Cj0262c.). The expression of proteins or polypeptides were analyzed by SDS-PAGE and Western blotting with an anti-His monoclonal antibody (Supplementary Figure S1). Then, we detected the interaction between Cj0371 and these chemotaxis proteins by GST pull-down analysis. The results showed that Cj0371 only interacts with $\mathrm{CheV}$. No interaction was detected with CheA, CheY, Cj1110c, and other polypeptides (Figures 2A,B). Other screened chemotaxis

TABLE 2 | Proteins that probably interact with the Cj0371 proteins identified in this study.

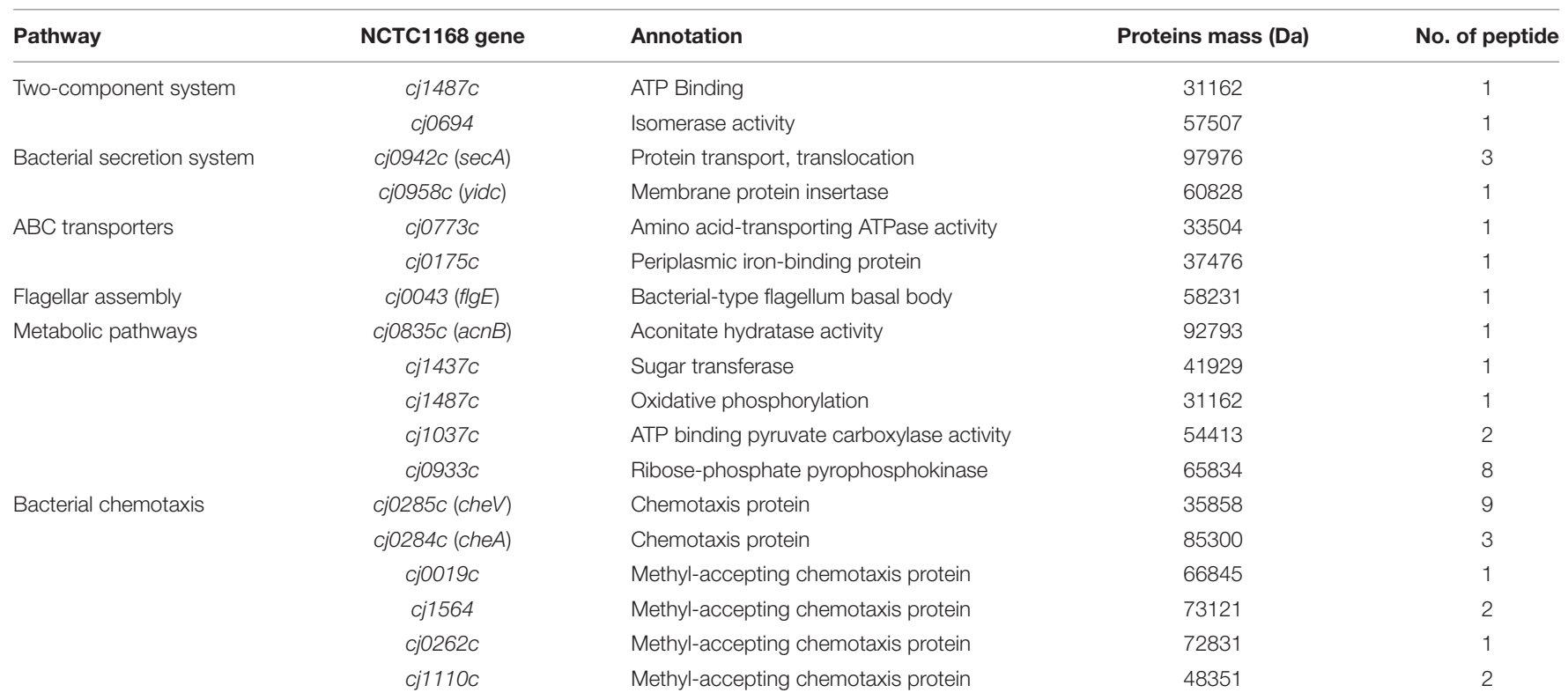


A

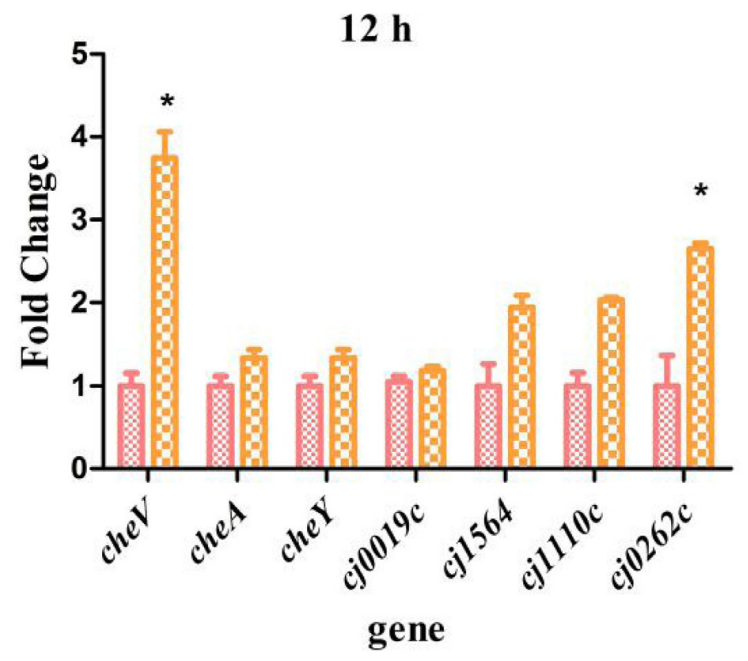

C

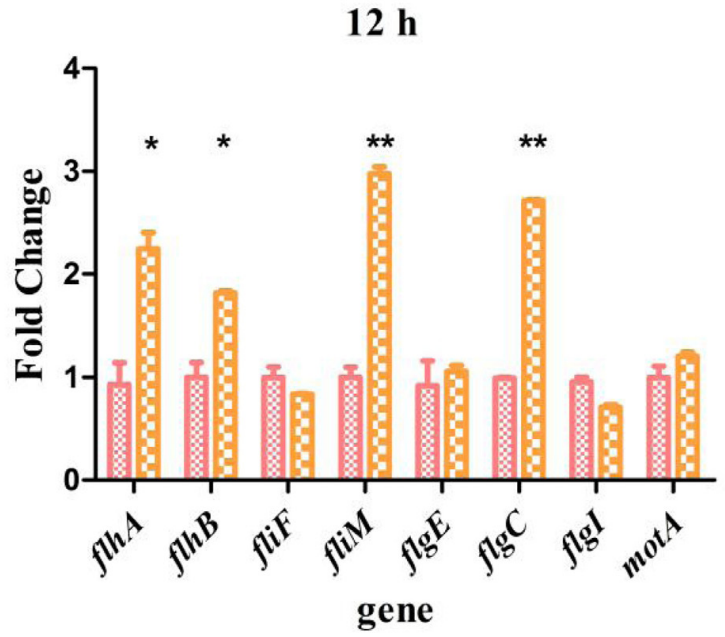

B

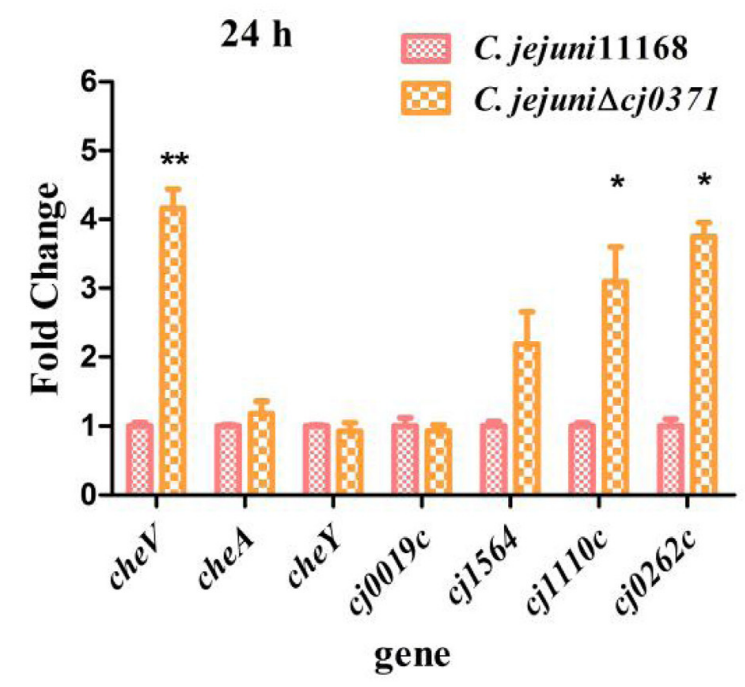

D

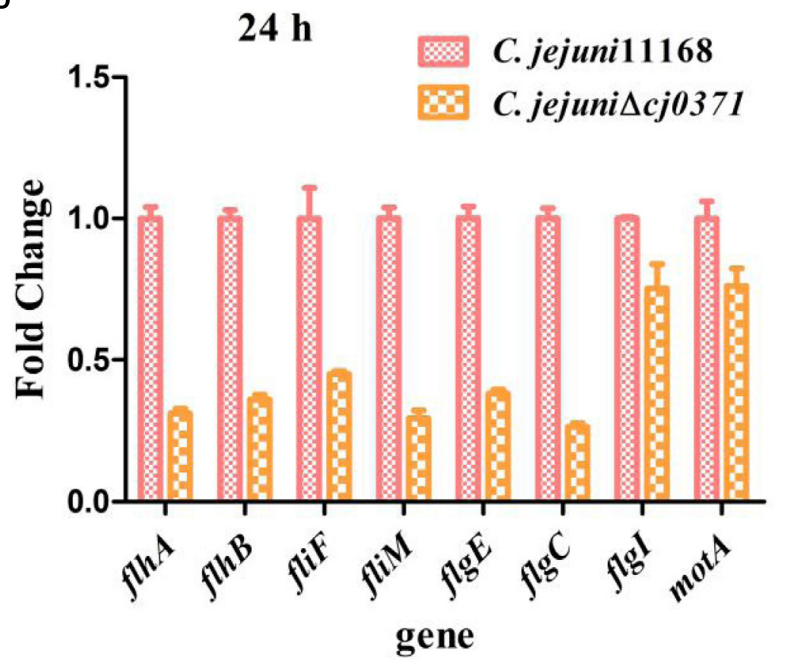

FIGURE 1 | Analysis of the relative expression of chemotaxis genes and basal flagellar genes by real-time PCR. The relative expression of major chemotaxis pathway genes (including these chemotaxis genes that screened by co-IP) at $12 \mathrm{~h}$ of $\mathrm{C}$. jejuni growth (A) and stationary phase (24 h) (B). (C,D) Show the relative expression of flagellar base genes at $12 \mathrm{~h}$ and $24 \mathrm{~h}$ of $C$. jejuni growth. C. jejuni RNA was reverse-transcribed to CDNA, and the expression values of all chemotaxis genes and flagellar genes are represented as relative to wild-type levels and normalized to 16S RNA. Mean values and standard deviations from triplicate measurements are shown. Significant $P$-values are indicated by asterisks (Student's $t$-test, unpaired, one-sided) as follows: ${ }^{*} 0.01<P<0.05 ;{ }^{* *} 0.001<P<0.01$.

proteins were identified to be false positive proteins from the co-IP analysis. When the washing strength of co-IP analysis was weak, many cascade proteins were eluted with first protein that interacted with $\mathrm{Cj0371.} \mathrm{The} \mathrm{screened} \mathrm{false} \mathrm{positive} \mathrm{proteins} \mathrm{are}$ not useless because by assessing the proteins they are in the same pathway, we can infer the possible pathways in which unknown proteins may play a role.

CheV has two domains: the CheW-like domain and the receiver (REC) domain. The $\mathrm{CheW}$-like domain is crucial for physically connecting chemoreceptors to the CheA kinase, and phosphorylation of the $\mathrm{CheV}$ receiver domain might adjust the efficiency of its coupling and thus allow the system to modulate the response to chemical stimuli in an adaptation process (Alexander et al., 2010). We also used the DE3 (pET-19b) expressing system to express the two domains of $\mathrm{CheV}$, and used GST pull-down to detect which domain interacts with Cj0371. The results showed that Cj0371 interacts with the regulatory domain of $\mathrm{CheV}$ rather than the $\mathrm{CheW}$-like domain (Figure 2B). Therefore we inferred that $\mathrm{Cj} 0371$ may regulate the receiver (REC) domain of $\mathrm{CheV}$ to influence chemotaxis.

\section{Cj0371 Inhibits the ATPase Activity of CheA}

CheA is a chemotaxis histidine kinase. It contains the known CheA functional domains: a phosphorylation site (P1 domain), 


\section{A}

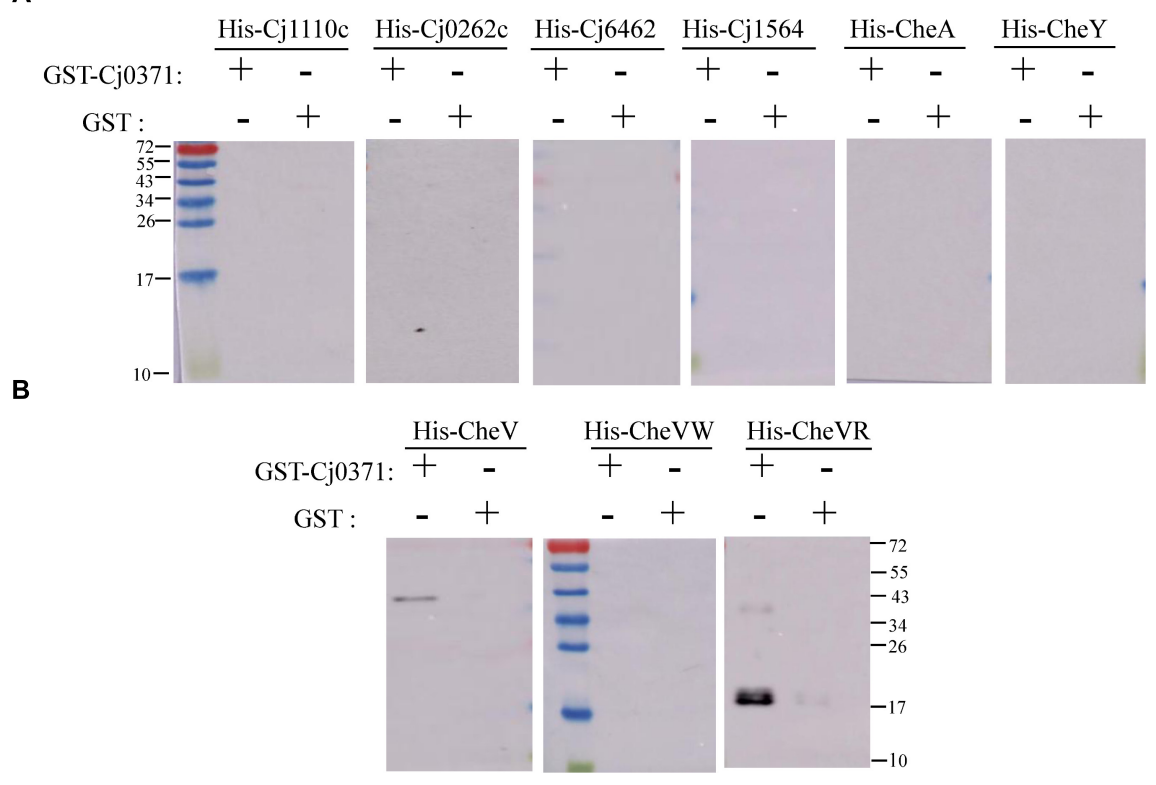

FIGURE 2 | Confirmation of interactions between chemotaxis proteins and Cj0371 by Western blot analysis. No interaction was detected between Cj0371 and CheA, CheY, Cj1110c and other polypeptides (A). The interaction between Cj0371 and CheV, the regulatory domain and the CheW-like domain of CheV (B). The size of the pre-stain marker is marked in numbers, and the unit is kDa. His-tagged chemotaxis proteins were co-eluted with GST-tagged Cj0371 when assays, described in the experimental procedures, were performed with cell lysates overexpressing both fusion proteins. His-tagged proteins expressed by DE3 (pET-19b) were analyzed by Western blotting using an anti-His antibody.

a catalytic kinase domain, a receptor-docking region (Bilwes et al., 1999). We purified chemotaxis proteins and Cj0371 (Supplementary Figure S2), then simulated the chemotaxis system of C. jejuni in vitro and detected the ATPase activity of CheA with and without Cj0371 protein in the chemotaxis reaction medium. The results showed that the reaction curve is broadly horizontal when any protein was in the basic medium (red line; Figure 3), this is an important negative control to ensure that changes in the mix are caused by chemotaxis proteins. The expected decreases were rapidly generated when the chemotaxis protein mixes were added to the reaction. In the absence of $\mathrm{Cj0371,} \mathrm{the} \mathrm{rate} \mathrm{of} \mathrm{NADH}$ decrease is faster than when Cj0371 was added to the reaction, which demonstrates that Cj0371 influences the ATPase activity of CheA and that the influence is negative.

\section{DISCUSSION}

C. jejuni $\Delta c j 0371$ displays hypermotility, enhanced growth kinetics, and increased invasion and colonization ability. It is interesting to note that cj0371 influences C. jejuni chemotaxis. Bacteria motility is not only regulated by flagella but also controlled by chemotactic factors. Bacteria use a signaling cascade of protein phosphorylation and dephosphorylation reactions to control bacterial motors in response to environmental chemical changes (Miller et al., 2009). Therefore, we speculated the increased chemotaxis may result from other changes in the cj0371 mutant. Based on

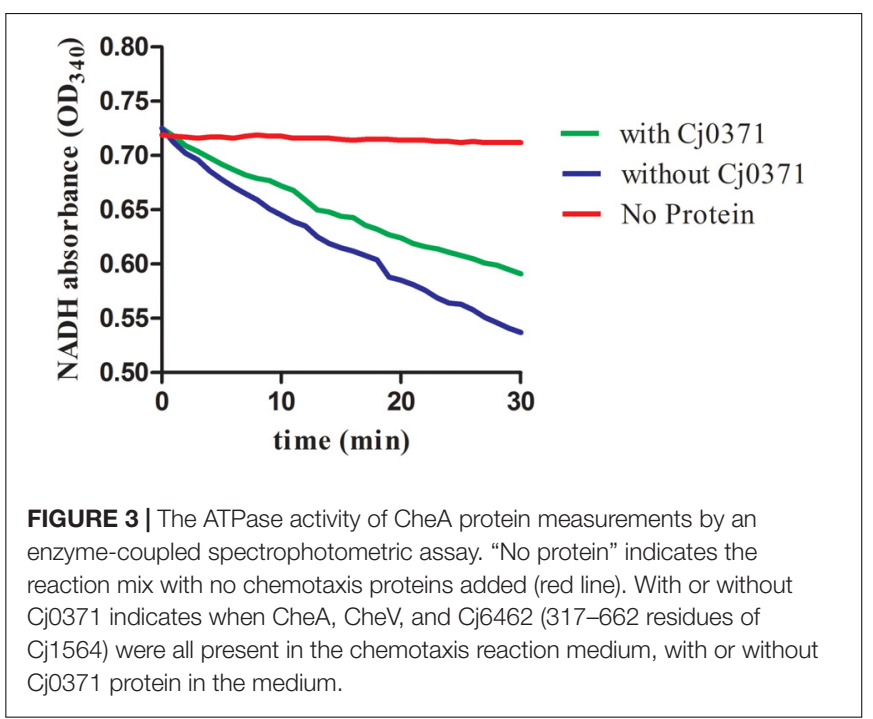

of previous research, this study continued to investigate the function of the cj0371 gene. Due to weak washing intensity in the co-IP experiments, approximately 20 putative proteins were screened, but not all of the screened proteins interact with Cj0371. Interestingly, there were six chemotaxis proteins in these screened proteins, including CheA, an important histidine kinase that receives and conducts signals in the chemotaxis pathway, CheV a scaffold protein that assists in signal conduction, as well as $\mathrm{Cj} 1564, \mathrm{Cj0262c}, \mathrm{Cj0019c}$, and $\mathrm{Cj} 1110 \mathrm{c}$, which are all MCP 


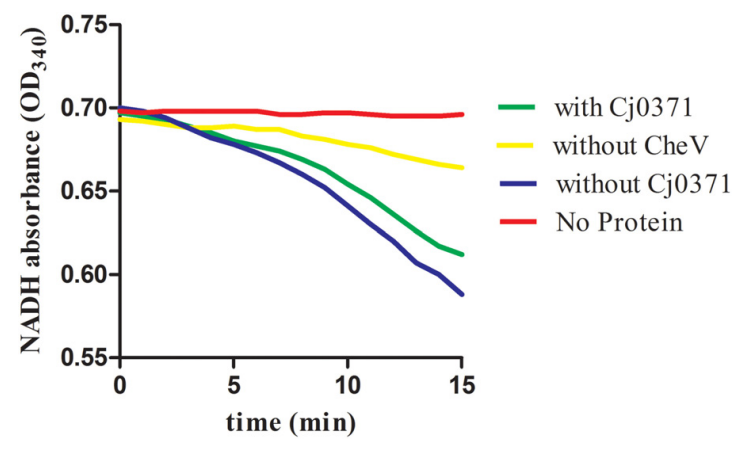

FIGURE 4 | The ATPase activity of CheA protein measured by an enzyme-coupled spectrophotometric assay. With or without CheV indicates that CheA, CheV, Cj6462, and Cj0371 were all in the chemotaxis reaction, with or without CheV protein in the medium.

proteins that sense environmental signal. In the chemotaxis pathway, these six chemotaxis proteins are all important component. Using the cheV isogenic mutants in the nutrientdepleted chemotaxis assay, it showed significantly reduced chemotaxis compared with wild-type cells toward L-serine $(P<0.05)$, and the cheV isogenic mutants also $(9.3 \mathrm{~mm} \pm 2.2$; $P<0.05)$ displayed significantly reduced motility compared with C. jejuni 11168-O wild-type (19.2 $\mathrm{mm} \pm 2.2)$ when examined in a $0.5 \%$ Brucella agar motility assay (Hartley-Tassell et al., 2010). In our experiments testing the ATPase activity of CheA protein measurements with an enzyme-coupled spectrophotometric assay, we found that when $\mathrm{CheV}$ protein was not present in the chemotaxis reaction, the rate of ATP hydrolysis is the slowest (yellow line; Figure 4). cj1110c functions through controlling energy taxis to keep signaling balanced in C. jejuni. Inactivation of the cj1110c gene, a cytoplasmic sensor with two PAS-domains, resulted in increased taxis (Reuter and van Vliet, 2013). An isogenic mutant of $c j 1564$ was shown to have altered phenotypic characteristics, including reduced chemotactic motility and reduced ability to adhere and invade a cultured epithelial cell line (Rahman et al., 2014). For the gene cj0262c, we did not find any report to explain the phenotypic characteristics of the mutant. These genes all had up-regulated expression due to the mutation of $c j 0371$. Therefore, we speculated that $c j 0371$ hinders chemotaxis of $C$. jejuni. The mechanism of chemotaxis control of bacteria flagella is highly complex, but it can be sure that chemotaxis directly adjusts the base of flagellar proteins such as FliM. Then, this study selected seven flagellar basic proteins of $C$. jejuni for analysis. In log phase growth of C. jejuni, fliM, $f l h A, f l h B$, and other genes, all showed up-regulated expression in $C$. jejuni $\Delta c j 0371$. The results of this analysis proved that the cj0371 gene influences the flagellar pathway of C. jejuni.

In this research, only $\mathrm{CheV}$ interacts with $\mathrm{Cj0371}$, other chemotaxis proteins were the false positive interactors from co-IP analysis. As previously reported, $\mathrm{CheV}$ interacts with residues 517-662 of Cj1564 (Tlp3), and this region is identical to residues 513-659 of Cj0144 (Tlp2) and 520-665 of Cj0262c (Tlp4) (Rahman et al., 2014). The interaction between $\mathrm{CheV}$ and CheA was previously identified by a yeast three hybrid-analysis (Hartley-Tassell et al., 2010). In addition, the phosphorylation signal transduction cascade associated with the methyl-accepting chemotaxis protein (MCP) sensor-CheA-CheW-CheY-flagellar motor backbone is a common basic principle in the chemotaxis systems of C. jejuni (Gegner et al., 1992; Marchant et al., 2002). Therefore, the C. jejuni chemotaxis pathway is a complex protein network, and the proteins interact with each other (Figure 5A). Taking the interaction between $\mathrm{CheV}$ and $\mathrm{Cj0371}$ as the core interaction, it is not surprising that we screened other chemotaxis cascade-interactive proteins. Now, we can confirm that cj0371 plays a role in the chemotaxis pathway. However, how cj0371

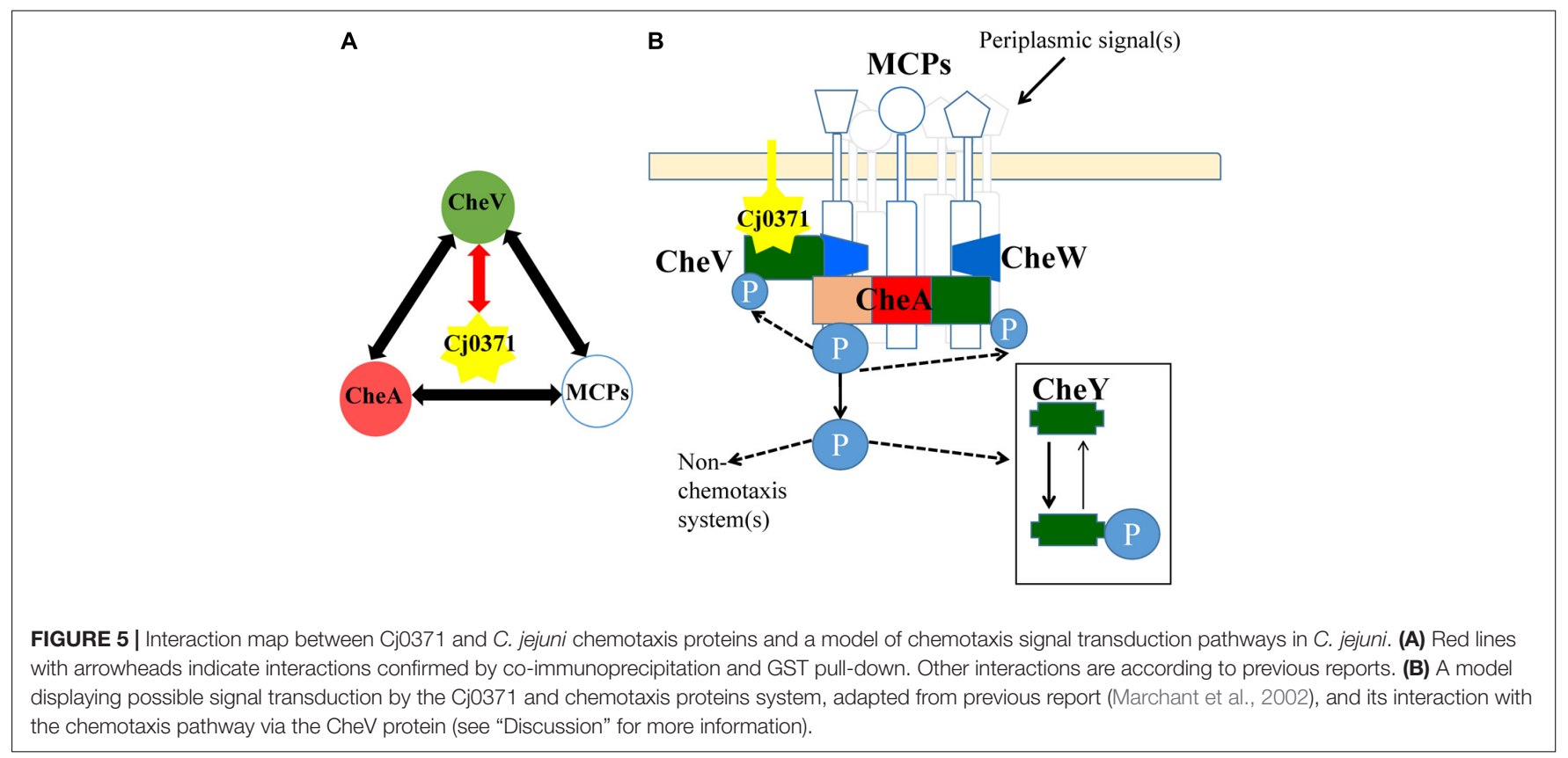


works in the chemotaxis pathway and what function it plays still need more research to confirm.

To thoroughly research the function of Cj0371 protein, we simulated the chemotaxis pathway of C. jejuni in vitro and assayed the ATPase activity of CheA with the Cj0371 protein present or absent. We found that Cj0371 inhibited CheA from hydrolyzing ATP in the reaction medium. cj0371 plays a negative role in the chemotaxis pathway. This result is consistent with our previous research showing that C. jejuni $\Delta c j 0371$ displays hyperchemotaxis, motility and so on.

Cj0371 interacts with $\mathrm{CheV}$, which is a central component of the C. jejuni chemotaxis signal transduction pathway. $\mathrm{CheV}$ consists of a CheW domain fused to a receiver domain that is capable of being phosphorylated. Further study showed that Cj0371 interacted with the receiver (REC) domain of CheV. The function of the $\mathrm{CheV}$ protein is not completely understood in C. jejuni (Zautner et al., 2012). However, we have confirmed that $\mathrm{CheV}$ plays a crucial role in the chemotaxis pathway. As the enzyme-coupled spectrophotometric assay showed, when $\mathrm{CheV}$ was not present in the chemotaxis reaction medium, the rate of ATP hydrolysis was almost zero (Figure 4). As the TMHMM ${ }^{1}$ database predicts, and consistent with the localization of cell cytoplasm and membrane analysis, Cj0371 is a transmembrane protein (Supplementary Figure S3). Phosphorylation of the $\mathrm{CheV}$ receiver domain might allow the chemotaxis pathway system to modulate the response to chemical stimuli in an adaptation process. We have shown that $\mathrm{Cj} 0371$ plays a role in the chemotaxis pathway. According our research and previous report model, we modified the model of $C$. jejuni chemotaxis pathway (Figure 5B). Whether Cj0371 protein through preventing $\mathrm{CheV}$ phosphorylation to decrease chemotaxis? Asp residue is important for the phosphorylation of regulators containing REC domain. In future studies, We will research the change of phosphorylation of 242 Asp residue (4-aspartylphosphate) when cj0371 gene was inactivated.

\section{CONCLUSION}

In conclusion, cj0371 plays a negative role in the chemotaxis of C. jejuni. It is not good for bacterial evolution to have one gene that is able to reduce virulence or inhibit the growth and metabolism of bacteria. Therefore, why is cj0371 stable and conserved in the genome of $C$. jejuni? In fact, some genes

${ }_{1}$ www.cbs.dtu.dk/services/TMHMM

\section{REFERENCES}

Alexander, R. P., Lowenthal, A. C., Harshey, R. M., and Ottemann, K. M. (2010). $\mathrm{CheV}$ : chew-like coupling proteins at the core of the chemotaxis signaling network. Trends Microbiol. 18, 494-503. doi: 10.1016/j.tim.2010.07.004

Bereswill, S., Alutis, M. E., Grundmann, U., Fischer, A., Gobel, U. B., and Heimesaat, M. M. (2016). Interleukin-18mediates immune responses to Campylobacter jejuni infection in gnotobiotic mice. PLoS One 11:e0158020. doi: 10.1371/journal.pone.0158020

Berradre-Saenz, B., Yanez-Ortega, J. L., Garcia-Sanchez, L., Melero-Gil, B., Rovira-Carballido, J., Carraminana-Martinez, I., et al. (2017). Epidemiology of are called 'anti-virulence' genes, and multiple hypotheses have been offered to explain why these genes are retained in the genome of bacteria (Foreman-Wykert and Miller, 2003; Rasko and Sperandio, 2010). Regarding the cj0371 gene, we prefer the view that it allows $C$. jejuni to evolve toward benign coexistence with its host, as occurs in poultry (it is well-known that poultry is an important reservoir of C. jejuni). Until now, there has been no explanation as to why $C$. jejuni can coexist with poultry. The function of this 'anti-virulence' gene may be an important reason.

Researching the function of new gene in the chemotaxis pathway, as described in this study, will contribute to understanding chemotaxis signaling pathways which are involved in colonization and its involvement in the chemotaxis pathway and its importance in the survival of this organism. This study adds a new component in the chemotaxis pathway of C. jejuni for the first time and provide the complexity framework for the full elucidation of the complex chemotaxis system of C. jejuni. The findings in this study also provide insight into the complexity of chemotaxis receptor protein-ligand interactions with implications not just for $\mathrm{C}$. jejuni chemotaxis but for all bacterial chemotaxis.

\section{AUTHOR CONTRIBUTIONS}

$\mathrm{XD}, \mathrm{JH}$, and $\mathrm{XJ}$ conceived and designed the experiments. $\mathrm{XD}$, $\mathrm{KK}, \mathrm{HT}$, and HYT performed the experiments. XD analyzed the data. XD, JH, and XJ contributed reagents, materials, and analysis tools. XD wrote the paper.

\section{FUNDING}

This work was supported by The National Key Research and Development Program of China (2018YFD0500500), National Key Technology R\&D Program (2014BAD13B02), Priority Academic Program Development of Jiangsu Higher Education Institutions, and the Six Talent Peaks Project in Jiangsu Province (2015-SWYY-02).

\section{SUPPLEMENTARY MATERIAL}

The Supplementary Material for this article can be found online at: https://www.frontiersin.org/articles/10.3389/fmicb. 2018.01904/full\#supplementary-material

campylobacteriosis in castile and leon, spain,during the period 2008-2015. Rev. Esp. Salud Publica 91:e201703030.

Bilwes, A. M., Alex, L. A., Crane, B. R., and Simon, M. I. (1999). Structure of CheA, a signal-transducing histidine kinase. Cell 96, 131-141. doi: 10.1016/S00928674(00)80966-6

Bronnec, V., Turonova, H., Bouju, A., Cruveiller, S., Rodrigues, R., Demnerova, K., et al. (2016). Adhesion, biofilm formation, and genomic features of Campylobacter jejuni Bf, an atypical strain able to grow under aerobic conditions. Front. Microbiol. 7:1002. doi: 10.3389/fmicb.2016.01002

Chandrashekhar, K., Kassem, I., Nislow, C., Gangaiah, D., Candelero-Rueda, R. A., and Rajashekara, G. (2015). Transcriptome analysis of Campylobacter 
jejuni polyphosphate kinase (ppk1 and ppk2) mutants. Virulence 6, 814-818. doi: 10.1080/21505594.2015.1104449

Du, X., Wang, N., Ren, F., Tang, H., Jiao, X., and Huang, J. (2016). cj0371: a novel virulence-associated gene of Campylobacter jejuni. Front. Microbiol. 7:1094. doi: 10.3389/fmicb.2016.01094

Faber, E., Gripp, E., Maurischat, S., Kaspers, B., Tedin, K., Menz, S., et al. (2016). Novel immunomodulatory flagellin-like protein FlaC in Campylobacter jejuni and other campylobacterales. mSphere 1:e00028-15. doi: 10.1128/mSphere. 00028-15

Flanagan, R. C., Neal-McKinney, J. M., Dhillon, A. S., Miller, W. G., and Konkel, M. E. (2009). Examination of Campylobacter jejuni putative adhesins leads to the identification of a new protein, designated FlpA, required for chicken colonization. Infect. Immun. 77, 2399-2407. doi: 10.1128/IAI.01266-08

Foreman-Wykert, A. K., and Miller, J. F. (2003). Hypervirulence and pathogen fitness. Trends Microbiol. 11, 105-108. doi: 10.1016/S0966-842X(03)00 007-6

Gao, B., Lara-Tejero, M., Lefebre, M., Goodman, A. L., and Galan, J. E. (2014). Novel components of the flagellar system in epsilonproteobacteria. mBio 5:e01349-14. doi: 10.1128/mBio.01349-14

Gegner, J. A., Graham, D. R., Roth, A. F., and Dahlquist, F. W. (1992). Assembly of an MCP receptor, CheW, and kinase CheA complex in the bacterial chemotaxis signal transduction pathway. Cell 70, 975-982. doi: 10.1016/0092-8674(92) 90247-A

Hartley-Tassell, L. E., Shewell, L. K., Day, C. J., Wilson, J. C., Sandhu, R., Ketley, J. M., et al. (2010). Identification and characterization of the aspartate chemosensory receptor of Campylobacter jejuni. Mol. Microbiol. 75, 710-730. doi: 10.1111/j.1365-2958.2009.07010.x

Helwigh, B., Porsbo, L. J., Boysen, L., and Bager, F. (2015). The European Union summary report on trends and sources of zoonoses, zoonotic agents and food-borne outbreaks in 2014. EFSA J. 13:4329.

Hoang, K. V., Stern, N. J., Saxton, A. M., Xu, F., Zeng, X., and Lin, J. (2011). Prevalence, development, and molecular mechanisms of bacteriocin resistance in Campylobacter. Appl. Environ. Microbiol. 77, 2309-2316. doi: 10.1128/AEM. 02094-10

Levit, M. N., Liu, Y., and Stock, J. B. (1998). Stimulus response coupling in bacterial chemotaxis: receptor dimers in signalling arrays. Mol. Microbiol. 30, 459-466. doi: 10.1046/j.1365-2958.1998.01066.x

Livak, K. J., and Schmittgen, T. D. (2001). Analysis of relative gene expression data using real-time quantitative PCR and the $2^{-\Delta \Delta C} \mathrm{~T}$ method. Methods 25, 402-408. doi: 10.1006/meth.2001.1262

Marchant, J., Wren, B., and Ketley, J. (2002). Exploiting genome sequence: predictions for mechanisms of Campylobacter chemotaxis. Trends Microbiol. 10, 155-159. doi: 10.1016/S0966-842X(02)02323-5

Miller, L. D., Russell, M. H., and Alexandre, G. (2009). Diversity in bacterial chemotactic responses and niche adaptation. Adv. Appl. Microbiol. 66, 53-75. doi: 10.1016/S0065-2164(08)00803-4

Nachamkin, I., Yang, X. H., and Stern, N. J. (1993). Role of Campylobacter jejuni flagella as colonization factors for three-day-old chicks: analysis with flagellar mutants. Appl. Environ. Microbiol. 59, 1269-1273.

Ninfa, A. J., Ninfa, E. G., Lupas, A. N., Magasanik, B., and Stock, J. (1988). Crosstalk between bacterial chemotaxis signal transduction proteins and regulators of transcription of the Ntr regulon: evidence that nitrogen assimilation and chemotaxis are controlled by a common phosphotransfer mechanism. Proc. Natl. Acad. Sci. U.S.A. 85, 5492-5496. doi: 10.1073/pnas.85.15.5492
Ninfa, E. G., Stock, A., Mowbray, S., and Stock, J. (1991). Reconstitution of the bacterial chemotaxis signal transduction system from purified components. J. Biol. Chem. 266, 9764-9770.

Rahman, H., King, R. M., Shewell, L. K., Semchenko, E. A., Hartley-Tassell, L. E., Wilson, J. C., et al. (2014). Characterisation of a multi-ligand binding chemoreceptor CcmL (Tlp3) of Campylobacter jejuni. PLoS Pathog. 10:e1003822. doi: 10.1371/journal.ppat.1003822

Rasko, D. A., and Sperandio, V. (2010). Anti-virulence strategies to combat bacteria-mediated disease. Nat. Rev. Drug Discov. 9, 117-128. doi: 10.1038/ $\operatorname{nrd} 3013$

Reuter, M., and van Vliet, A. H. (2013). Signal balancing by the CetABC and CetZ chemoreceptors controls energy taxis in Campylobacter jejuni. PLoS One 8:e54390. doi: 10.1371/journal.pone.0054390

Schieltz, D. M., Washburn, M. P., and Hays, L. G. (2006). Analysis of complex protein mixtures using Nano-LC coupled to MS/MS. Cold Spring Harb. Protoc. 2006:pdb.prot4553. doi: 10.1101/pdb.prot4553

Sehgal, P., Olesen, C., and Moller, J. V. (2016). ATPase activity measurements by an enzyme-coupled spectrophotometric assay. Methods Mol. Biol 1377, 105-109. doi: 10.1007/978-1-4939-3179-8_11

Sheng, J., Yu, H., Li, J., Sheng, G., Zhou, L., and Lu, Y. (2007). Cloning and expression of the human augmenter of liver regeneration at low temperature in Escherichia coli. J. Biochem. Biophys. Methods 70, 465-470. doi: 10.1016/j.jbbm. 2006.11.009

Suryanarayana, N., Vanlalhmuaka, Mankere, B., Verma, M., Thavachelvam, K., and Tuteja, U. (2016). Soluble expression and characterization of biologically active bacillus anthracis protective antigen in Escherichia coli. Mol. Biol. Int. 2016:4732791. doi: 10.1155/2016/4732791

Szymanski, C. M., King, M., Haardt, M., and Armstrong, G. D. (1995). Campylobacter jejuni motility and invasion of Caco-2 cells. Infect. Immun. 63, 4295-4300.

Vikis, H. G., and Guan, K. L. (2004). Glutathione-S-transferase-fusion based assays for studying protein-protein interactions. Methods Mol. Biol. 261, 175-186. doi: 10.1385/1-59259-762-9:175

Wakerley, B. R., Uncini, A., Yuki, N., and GBS Classification Group (2014). Guillain-barre and miller fisher syndromes-new diagnostic classification. Nat. Rev. Neurol. 10, 537-544. doi: 10.1038/nrneurol.2014.138

Welch, M., Oosawa, K., Aizawa, S., and Eisenbach, M. (1993). Phosphorylationdependent binding of a signal molecule to the flagellar switch of bacteria. Proc. Natl. Acad. Sci. U.S.A. 90, 8787-8791. doi: 10.1073/pnas.90.19.8787

Zautner, A. E., Tareen, A. M., Gross, U., and Lugert, R. (2012). Chemotaxis in Campylobacter jejuni. Eur. J. Microbiol. Immunol. 2, 24-31. doi: 10.1556/EuJMI. 2.2012.1.5

Conflict of Interest Statement: The authors declare that the research was conducted in the absence of any commercial or financial relationships that could be construed as a potential conflict of interest.

Copyright (c) 2018 Du, Kong, Tang, Tang, Jiao and Huang. This is an open-access article distributed under the terms of the Creative Commons Attribution License (CC BY). The use, distribution or reproduction in other forums is permitted, provided the original author(s) and the copyright owner(s) are credited and that the original publication in this journal is cited, in accordance with accepted academic practice. No use, distribution or reproduction is permitted which does not comply with these terms. 